\section{Clinical utilization of four-factor prothrombin complex concentrate: a retrospective single center study}

\author{
Zaina Naeem', Salsabeel Allan', Aneury Hernandez', Dennis K Galanakis², \\ Adam J. Singer ${ }^{1}$
}

'Department of Emergency Medicine, Renaissance School of Medicine at Stony Brook University, Stony Brook, NY, USA

${ }^{2}$ Department of Pathology, Renaissance School of Medicine at Stony Brook University, Stony Brook, NY, USA

Objective Four-factor prothrombin complex concentrate (4F-PCC) was approved by the US Food and Drug Administration in 2013 for management of severely bleeding patients on warfarin therapy. We describe use of 4F-PCC at a large, suburban academic center.

Methods We retrospectively reviewed all patients receiving 4F-PCC from its introduction through 2016 at a large level 1 trauma center. Clinical and demographic data were obtained, including indications for anticoagulation and antiplatelet agents, comorbidities, concomitant medications, etiology and site of bleeding, as well as disposition, length of stay, mortality, and thrombotic events.

Results One hundred eighty-four patients received 4F-PCC. Mean age was 72 years; $40.8 \%$ were female. Indications for 4F-PCC administration included: active bleeding (74\%), reversal prior to a procedure (14\%), and elevated international normalized ratio (12\%). Warfarin was the most common concomitant medication (71.1\%). Most patients were receiving anticoagulation for atrial fibrillation (63\%). Concomitant treatments for bleeding included vitamin K (58.2\%), packed red blood cells (50\%), fresh frozen plasma (38\%), and platelets (26.1\%), amongst others. Median length of hospital stay was 8.4 days. Nine patients (4.9\%) developed thrombosis within 90 days of 4F-PCC. Mortality was $24.5 \%$, with notably higher rates amongst those who received 4F-PCC for off-label indications (19.1\% on-label mortality vs. $37.7 \%$ off-label mortality on chisquare analysis, $P=0.01$ ).

Conclusion This study demonstrates that 4F-PCC is being utilized for indications other than the reversal of warfarin-induced coagulopathy. Further investigation is warranted to determine the efficacy and safety of 4F-PCC for these potential indications.

Keywords Prothrombin complex concentrate; Anticoagulants; Blood coagulation factors; Vita$\min \mathrm{K}$

\section{elSSN: 2383-4625}

Received: 18 February 2020

Revised: 16 June 2020

Accepted: 19 June 2020

Correspondence to: Adam J. Singer Department of Emergency Medicine, Stony Brook University, HSC-L4-050, Stony Brook, NY 11794, USA

E-mail: Adam.singer@stonybrook.edu ORCID

https://orcid.org/0000-0003-4694-6152

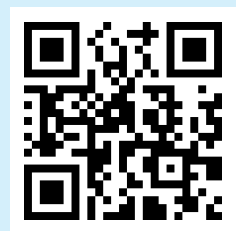

Naeem Z, Allan S, Hernandez A, Galanakis DK, Singer AJ. Clinical utilization of fourfactor prothrombin complex concentrate: a retrospective single center study. Clin Exp Emerg Med 2021;8(2):75-81. https://doi. org/10.15441/ceem.20.017

This is an Open Access article distributed under the terms of the Creative Commons Attribution Non-Commercial License (https:// creativecommons.org/licenses/by-nc/4.0/). 


\section{INTRODUCTION}

Warfarin, an oral anticoagulant, is commonly used for the prevention or treatment of thrombotic and embolic conditions, including atrial fibrillation, pulmonary embolism, and deep venous thrombosis. 'Warfarin is a vitamin $\mathrm{K}$ antagonist with a narrow therapeutic window and variable individual-specific dosing, and as such, carries a significant risk of bleeding, with reported rates ranging from $0 \%$ to $10 \% .^{1-3}$ Reversal of warfarin-associated bleeding has traditionally been accomplished using vitamin $\mathrm{K}$ or fresh frozen plasma (FFP) due to the widespread availability and relatively low cost of these agents. ${ }^{4}$ Disadvantages to using FFP include the need for ABO compatibility testing prior to administration, large volume requirements of up to $10-15 \mathrm{~mL} / \mathrm{kg}$, and prolonged thawing time. ${ }^{4-6}$

For these reasons, recombinant vitamin K-dependent factors and 3- or 4-factor prothrombin complex concentrates (4F-PCCs) have been increasingly utilized on or off-label for the reversal of warfarin-associated bleeding. 4F-PCC (Kcentra; CSL Behring, King of Prussia, PA, USA), which gained US Food and Drug Administration approval in 2013, is a blood coagulation factor replacement product containing the vitamin- $K$ dependent coagulation factors $\mathrm{II}, \mathrm{VII}, \mathrm{IX}, \mathrm{X}$, proteins $\mathrm{C}$ and $\mathrm{S}$, antithrombin III, and human albu$\mathrm{min}^{7.8}$ It is the only drug to be approved to date for the rapid reversal of warfarin in the setting of acute major bleeding or urgent need for surgical or invasive procedures.?

Despite its rising popularity, few studies have reported indications and outcomes with use of $4 \mathrm{~F}-\mathrm{PCC}$, and those that do focus on niche patient populations or specific dosing regimens. ${ }^{9-11}$ In our study, we describe use of 4F-PCC in the emergency department at a large, suburban academic center to elucidate practice patterns.

\section{METHODS}

\section{Study design}

We conducted a structured retrospective chart review of all adult patients receiving 4F-PCC from when it became available to our hospital in 2015 through 2016. Data were obtained from electronic medical records reviewed in Cerner PowerChart, the centralized clinical database for our hospital. Three independent reviewers extracted data to ensure reliable capturing; any discrepancies were resolved via discussion amongst the reviewers. Medical record review was consistent with the recommendations of Gilbert et al., ${ }^{12}$ as well as that of Kaji et al. ${ }^{13}$ Our study also followed the Strengthening of Reporting of Observational Studies in Epidemiology (STROBE) reporting guidelines for cross-sectional studies (http://www.equator-network.org/reporting-guidelines/ strobe/). Because of its retrospective design, this study was approved by our institutional review board (2017-4079) with waiver of informed consent.

\section{Setting}

The study was conducted at the emergency department of a university affiliated, tertiary care, suburban hospital in the northeast region of the US with an annual census of approximately 110,000 patients.

\section{Data measures}

Demographic and clinical data points of interest included indications for anticoagulation at baseline, concomitant anti-thrombotic use (agents of interest were warfarin, aspirin, clopidogrel, and direct oral anticoagulants), presence of bleed (and if present, bleed type and site of bleeding), and indications for reversal of anticoagulation with 4F-PCC. 4F-PCC use was considered "on-label" if it was administered for warfarin reversal in patients with either acute major bleeding or requiring urgent surgery/invasive procedure. ${ }^{7}$ Any use of 4F-PCC outside this scope was deemed off-label.

\section{Study outcomes}

The primary outcome of interest was the incidence of mortality, defined as failure to survive to hospital discharge, in patients receiving 4F-PCC for an on-label versus off-label indication. Secondary outcomes included patient disposition, length of hospital stay, and subsequent thrombotic event within 90 days of $4 \mathrm{~F}-\mathrm{PCC}$ administration. A 90-day assessment period was chosen in line with the cut-off designated by prior studies to facilitate capturing of long-term sequelae of reversal. ${ }^{6}$

\section{Data analysis}

Data were input into the REDCap electronic data capture system and summarized with descriptive and comparative statistics. Categorical data were summarized as frequencies and percentages. Continuous data were summarized as means and standard deviations. Patient subgroups were compared using chi-square statistics. The level of significance was set as a P-value of $<0.05$.

\section{RESULTS}

One hundred eighty-four patients received 4F-PCC during the study period. Mean \pm standard deviation age was $72 \pm 17$ years; $40.8 \%$ were female. Of all patients, $73.9 \%$ presented to the emergency department with a bleed, of which $36.8 \%$ were traumatic in etiology. The most common bleeding sites were intracra- 


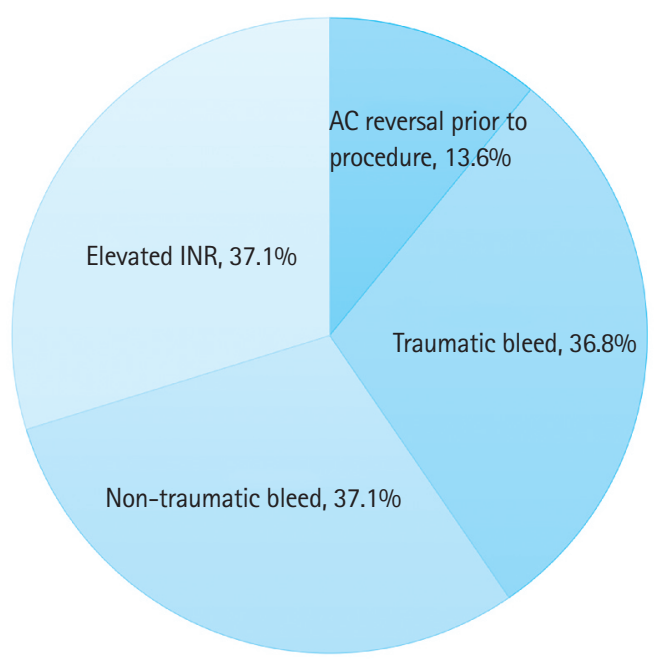

Fig. 1. Indications for 4-factor prothrombin complex concentrate. INR, international normalized ratio; $A C$, anticoagulation.

nial (28.8\%), lower gastrointestinal (15.2\%), upper gastrointestinal $(10.3 \%)$, hemothorax (3.3\%), abdomen (3.3\%), retroperitoneal (3.8\%), extremities (22\%), pelvic (2.2\%), and epistaxis $(2.2 \%)$. The remaining 48 patients presenting with no bleed required reversal for an upcoming procedure (52.1\%) or for an elevated international normalized ratio (INR, 47.9\%). For patients with traumatic events, the most common bleeding sites were intracranial (60\%), hemothorax (12\%), and abdominal bleeds (10\%). Indications for 4F-PCC administration are depicted pictorially in Fig. 1.

Concomitant medications of interest to this study included warfarin, aspirin, clopidogrel, and direct oral anticoagulants; data for each was extracted for the patient cohort. The majority of patients were on warfarin therapy (71.1\%), followed by aspirin (44.0\%), direct oral anticoagulants (15.2\%), and clopidogrel (9.2\%). Indications for anticoagulation included atrial fibrillation in $63.0 \%$ of patients, deep venous thrombosis in $15.8 \%$, pulmonary embolism in 6.5\%, prophylaxis for venous thromboembolism in $8.2 \%$ and mitral valve replacement in 3.3\%. $13.1 \%$ were on no anticoagulant prior to 4F-PCC administration. Concurrent treatments for bleeding included vitamin $\mathrm{K}(58.2 \%)$, packed red blood cells (50\%), FFP (38\%), platelets (26.1\%), factor VII (2.2\%), factor IX (0.5\%), and cryoprecipitate (8.2\%). Interventions included surgery in $27.2 \%$ and interventional radiology in $8.8 \%$ of patients. Patient demographics are outlined in Table 1.

Median (interquartile range) length of hospital stay was 8.4 (4.2-16.9) days. Nine patients (4.9\%) developed thrombosis within 90 days of $4 \mathrm{~F}-\mathrm{PCC}$; four developed deep vein thrombosis, two developed pulmonary emboli, one developed stroke, one developed thrombosis of a stent, and one developed acute thrombus of the left radial artery. In-hospital mortality was $24.5 \%$ across the
Table 1. Demographics of patients receiving 4F-PCC in the emergency department during the study period

\begin{tabular}{|c|c|}
\hline Demographics & Value \\
\hline No. of patients & 184 \\
\hline Age (yr) & $72 \pm 17$ \\
\hline Sex, male & $109(59)$ \\
\hline Sex, female & $75(41)$ \\
\hline \multicolumn{2}{|l|}{ Selected concomitant medications } \\
\hline Warfarin & $131(71)$ \\
\hline Aspirin & $81(44)$ \\
\hline Direct oral anticoagulants & $28(15)$ \\
\hline Clopidogrel & $17(9)$ \\
\hline Not on anticoagulant prior to $4 \mathrm{~F}-\mathrm{PCC}$ administration & $24(13)$ \\
\hline \multicolumn{2}{|l|}{ Indications for anticoagulation } \\
\hline Atrial fibrillation & $116(63)$ \\
\hline Deep venous thrombosis & $29(16)$ \\
\hline Venous thromboembolism prophylaxis & $15(8)$ \\
\hline Pulmonary embolism & $12(7)$ \\
\hline Mitral valve replacement & $6(3)$ \\
\hline \multicolumn{2}{|l|}{ Site of bleeding } \\
\hline Intracranial & $53(29)$ \\
\hline Lower gastrointestinal & $28(15)$ \\
\hline Upper gastrointestinal & $19(10)$ \\
\hline Retroperitoneal & $7(4)$ \\
\hline Hemothorax & $6(3)$ \\
\hline Extremities & $4(2)$ \\
\hline Abdominal & $6(3)$ \\
\hline Pelvic & $4(2)$ \\
\hline Epistaxis & $4(2)$ \\
\hline \multicolumn{2}{|l|}{ Concomitant treatments for bleeding } \\
\hline Vitamin K & $107(58)$ \\
\hline Packed red blood cells & $92(50)$ \\
\hline Fresh frozen plasma & $70(38)$ \\
\hline Platelets & $48(26)$ \\
\hline Cryoprecipitate & $15(8)$ \\
\hline Factor VII & $4(2)$ \\
\hline Factor IX & $1(1)$ \\
\hline \multicolumn{2}{|l|}{ Interventions } \\
\hline Surgery & $50(27)$ \\
\hline Interventional radiology & $16(9)$ \\
\hline
\end{tabular}

Values are presented as mean \pm standard deviation or number $(\%)$ unless otherwise indicated.

4F-PCC, 4-factor prothrombin complex concentrate.

entire patient cohort. Mortality was higher for patients who received 4F-PCC for off-label indications (19.1\% on-label mortality vs. $37.7 \%$ off-label mortality, $\mathrm{P}=0.01$ ) (Fig. 2). A comparison of patients treated on- and off-label is presented in Table 2.

\section{DISCUSSION}

The principal findings of this retrospective chart review of adult patients receiving 4F-PCC in the emergency department were (1) 


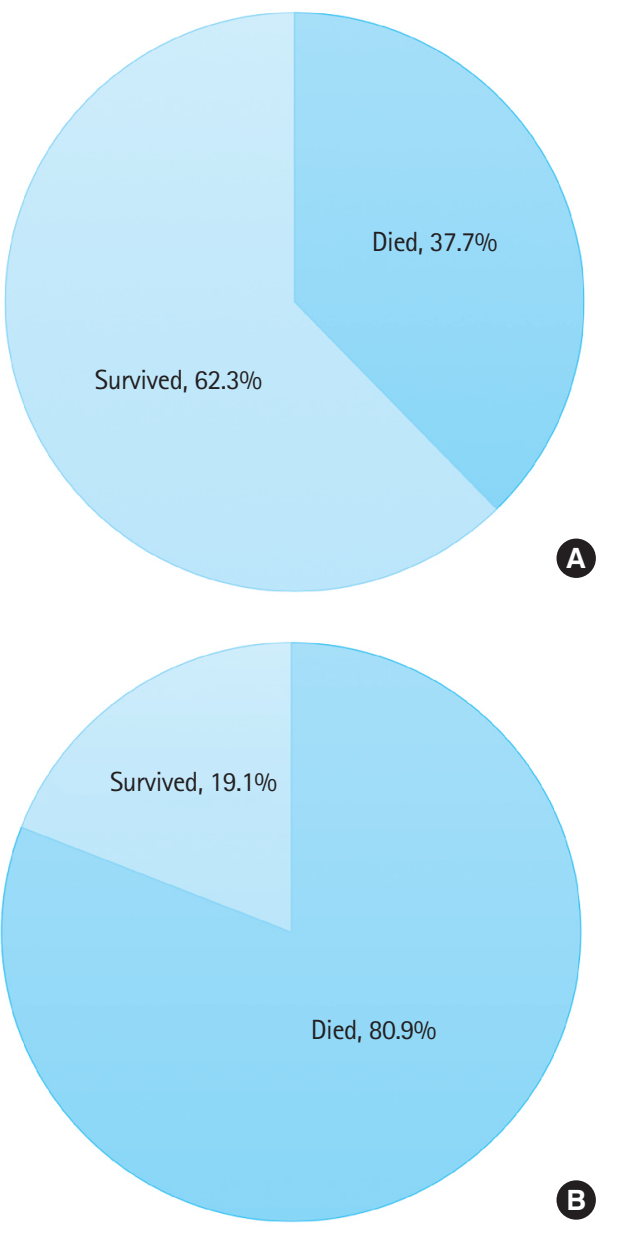

Fig. 2. Mortality by indication for 4-factor prothrombin complex concentrate. (A) Off-label and (B) on-label use of 4-factor prothrombin complex concentrate.

the most common indication for administration was active bleeding, with traumatic and spontaneous bleeds being equally likely; (2) overall mortality rate was 24.6\%; (3) there was an approximately two-fold increase in mortality for off-label use compared to on-label use of 4F-PCC; and (4) thromboembolic events (TEE) constituted a small but clinically relevant complication following 4F-PCC.

Reported mortality rates associated with $4 \mathrm{~F}-\mathrm{PCC}$ administration vary widely in the literature and may be attributed to the disease specific state of these cohort analyses. Huang et al.., ${ }^{14}$ for example, describe a mortality rate of $51.6 \%$ in patients with liver pathologies compared to $18.5 \%$ in non-liver disease patients. Fischer et al. ${ }^{15}$ report a lower in-hospital mortality of $25 \%$ following reversal of warfarin-associated intracranial hemorrhage with 3F-PCC or 4F-PCC, with more deaths in the 4F-PCC subset. We comparably demonstrate that $75.4 \%$ of our patient cohort, comprised solely of 4F-PCC recipients, survived to hospital dis-
Table 2. Comparison of on label and off label use of 4-factor prothrombin complex concentrate

\begin{tabular}{|c|c|c|c|}
\hline & $\begin{array}{l}\text { On label } \\
(n=131)\end{array}$ & $\begin{array}{c}\text { Off label } \\
(n=53)\end{array}$ & P-value \\
\hline Sex, male & $78(60)$ & $31(58)$ & 1.00 \\
\hline Age (yr) & $77 \pm 11$ & $60 \pm 22$ & $<0.001$ \\
\hline \multicolumn{4}{|l|}{ Antiplatelets } \\
\hline Aspirin & $67(51)$ & $14(26)$ & 0.003 \\
\hline Clopidogrel & $16(12)$ & $1(2)$ & 0.03 \\
\hline \multicolumn{4}{|l|}{ Comorbidities } \\
\hline Hypertension & $101(77)$ & $31(58)$ & 0.02 \\
\hline Diabetes & $47(36)$ & $12(23)$ & 0.12 \\
\hline Liver disease & $6(5)$ & $10(19)$ & 0.004 \\
\hline CKD & $36(27)$ & $6(11)$ & 0.02 \\
\hline Alcohol abuse & $3(2)$ & $6(11)$ & 0.02 \\
\hline Prior bleeding episode & $15(11)$ & $5(9)$ & 0.80 \\
\hline Prior stroke & $14(11)$ & $5(9)$ & 1.00 \\
\hline \multicolumn{4}{|l|}{ Bleed site } \\
\hline Intracranial & $38(29)$ & $15(28)$ & 1.00 \\
\hline Upper Gl & $13(10)$ & $6(11)$ & 0.79 \\
\hline Lower GI & $22(17)$ & $6(11)$ & 0.50 \\
\hline Epistaxis & $3(2)$ & 1 (92) & 1.00 \\
\hline Hemothorax & $5(4)$ & $1(2)$ & 0.68 \\
\hline Pelvic & $3(2)$ & $1(2)$ & 1.00 \\
\hline Abdominal & $3(2)$ & $3(6)$ & 0.36 \\
\hline Retroperitoneal & $5(4)$ & $2(4)$ & 1.00 \\
\hline Extremities & $0(0)$ & $4(8)$ & 0.006 \\
\hline Traumatic bleed & $33(34)$ & $17(44)$ & 0.33 \\
\hline \multicolumn{4}{|l|}{ Treatments } \\
\hline Vitamin K & $92(70)$ & $15(28)$ & $<0.001$ \\
\hline FFP & $44(34)$ & $26(49)$ & 0.07 \\
\hline PRBC & $63(48)$ & $29(55)$ & 0.52 \\
\hline Platelets & $23(18)$ & $26(47)$ & $<0.001$ \\
\hline Factor IX & $1(1)$ & $0(0)$ & 1.00 \\
\hline Factor VII & $0(0)$ & $4(8)$ & 0.006 \\
\hline Cryoprecipitate & $5(4)$ & $10(19)$ & 0.002 \\
\hline \multicolumn{4}{|l|}{ Interventions } \\
\hline None & $85(65)$ & $26(49)$ & 0.07 \\
\hline Surgery & $31(24)$ & $19(36)$ & 0.10 \\
\hline Interventional radiology & $9(7)$ & 7 (13) & 0.25 \\
\hline Hospital length of stay & $15 \pm 27$ & $20 \pm 26$ & 0.31 \\
\hline In-hospital mortality & 25 (19) & $20(38)$ & 0.01 \\
\hline
\end{tabular}

Values are presented as number (\%) or mean \pm standard deviation.

CKD, chronic kidney disease; GI, gastrointestinal; FFP, fresh frozen plasma; PRBC, packed red blood cells.

charge. In contrast, Berndtson et al. ${ }^{16}$ describe an in-hospital mortality rate of $0 \%$ and no thromboembolic complications in patients receiving 4F-PCC for reversal of warfarin-induced coag- 
ulopathy, though their sample size was relatively small at 26 patients.

While population-specific findings are useful for determining safety and efficacy data for a respective cohort of patients, these analyses fail to comprehensively demonstrate how 4F-PCC is being utilized by providers in practice, hence limiting our understanding of the potential scope of indications and associated outcomes for this relatively novel agent. Here, we characterize 4F-PCC use across all adult patients, irrespective of existing patient comorbidities, in the emergency department setting, which often serves as the initial point-of-contact for patients with acute bleeds or requiring urgent surgery.

Even though 4F-PCC has been marketed in Europe as Beriplex P/N (CSL Behring, Marburg, Germany) for a number of years, it only gained US Food and Drug Administration approval in 2013 for reversal in the setting of an acute major bleed or urgent surgical or invasive procedure. ${ }^{17}$ This study evaluates mortality rates for patients receiving 4F-PCC for on- versus off-label indications, including traumatic and spontaneous bleeds outside the scope of a "major bleed." Acute major bleeding was defined by Sarode et al. $^{8}$ in their phase Illb study of 4F-PCC as one of the following: life-threatening or potentially life-threatening (according to the treating physician); acute bleeding associated with a fall in hemoglobin $\geq 2 \mathrm{~g} / \mathrm{dL}$; and bleeding requiring blood product transfusion. We analyzed patient charts to correlate these parameters with time of 4F-PCC administration and found inconsistent reporting, necessitating future exploration of whether and how bleed severity is being considered by physicians in their decisionmaking to utilize this drug for reversal. In our study, the most common bleeding sites were intracranial and gastrointestinal, occurring in over one half of those presenting with an acute bleed. Interestingly, we found that amongst those presenting with no bleed, roughly half were reversed for the off-label indication of an elevated INR without an impending urgent surgical or invasive procedure.

To our knowledge, this is the first study to describe a nearly two-fold increase in mortality amongst patients receiving 4F-PCC for off-label indications. It is likely that those who received 4FPCC off-label were administered the drug once other therapeutic interventions had been exhausted; these patients were probably sicker and more likely to die. Additionally, a subset of our patients underwent surgery (27.2\%) and interventional radiology (8.8\%). These procedures carry their own inherent complication risk, including mortality, potentially adversely affecting patient outcomes. Future analyses stratifying patients per comorbidity would allow us to appreciate risk factors for 4F-PCC-associated mortality. There remains a need for randomized controlled trials targeted towards these subpopulations to inform potential expansion of indications for 4F-PCC in the clinical setting.

TEE have been described as a serious concern following 4F-PCC administration. Sin et al. ${ }^{18}$ report a rate of $11.8 \%$ of TEE within 14 days following 4F-PCC administration, with significant risk factors including heparin-induced thrombocytopenia, major surgery within 14 days, and the presence of greater than six thrombotic risk factors. Joseph et al. ${ }^{19}$ similarly describe TEE in 6.2\% of patients, with factor $V$ Leiden or antiphospholipid syndrome being predictive on multivariate analysis. Our findings echo those in the literature. While we do not elucidate risk factors for TEE, we describe TEE incidence of $4.9 \%$ within 90 days of $4 F-P C C$, with deep vein thrombosis constituting approximately half of these events. A prior case report of 3F-PCC has described new limb-threatening deep vein thrombosis as a complication of 3F-PCC, and our findings suggest that this complication may also develop in $4 \mathrm{~F}$ PCC treated patients, likely due to shared mechanisms related to factor replacement in the coagulation cascade as well as patients' underlying thrombotic risk. ${ }^{20}$ It has been postulated that the higher factor VII content of 4F-PCC compared to 3F-PCC puts patients at higher risk for clot development. ${ }^{1}$

Additional complications associated with 4F-PCC include INR rebound and rebleeding, and it has been suggested that intravenous vitamin $\mathrm{K}$ be given if a sustained reduction in INR is desired. ${ }^{18}$ Indeed, we report that vitamin $\mathrm{K}$ was the most common concurrent treatment for bleeding, followed by packed red blood cells, FFP, and platelets. Most patients in our study were on warfarin therapy, which was expected as 4F-PCC is specifically indicated for warfarin reversal. Approximately one-tenth of our cohort was on a direct oral anticoagulant prior to 4F-PCC administration. Studies suggest that while 4F-PCC may be a promising candidate for the reversal of rivaroxaban and apixaban, data supporting this clinical utilization is lacking. ${ }^{21}$ Direct oral anticoagulant reversal remains a potential area for expansion of $4 \mathrm{~F}-\mathrm{PCC}$ clinical application.

Our study has several limitations. It is a single-institution retrospective chart review at an academic suburban center. Findings in our study may not be generalizable to patient populations who reside elsewhere. While our findings point to higher rates of mortality for patients receiving 4F-PCC for an off-label indication, our study is not designed to establish a causal relationship between these variables.

As this is a descriptive study, we did not control for potential confounders, including existing comorbidities or the concomitant use of anticoagulant or antiplatelet drugs, which could have affected patient response to 4F-PCC and subsequent morbidity and mortality outcomes. Additionally, patients who were discharged 
from our emergency department following 4F-PCC administration and presented for thrombosis elsewhere were outside the scope of our electronic medical records and limited our ability to capture all adverse events following 4F-PCC administration.

This study assumes that the data points of interest were comprehensively and routinely recorded by healthcare practitioners in patient charts. During the review, inconsistencies were noted in how units of blood products were recorded both within a single chart and across multiple charts. We attempted to minimize discrepancies in data extraction and interpretation by appointing three independent reviewers, with inconsistencies resolved via discussion.

In conclusion, 4F-PCC is being utilized for indications other than the reversal of warfarin-induced coagulopathy, with higher mortality rates noted for the subset of patients receiving the agent for off-label indications. Further investigation in a controlled clinical setting is warranted to determine the efficacy and safety of 4F-PCC for such indications.

\section{CONFLICT OF INTEREST}

Adam J. Singer serves as Editor-in-Chief of Clinical and Experimental Emergency Medicine, but had no role in the decision to publish this article. AJS is a consultant and speaker for Alexion, Janssen, Pfizer, and BMS.

\section{REFERENCES}

1. Holt $T$, Taylor $S$, Abraham $P$, et al. Three- versus four-factor prothrombin complex concentrate for the reversal of warfarin-induced bleeding. Int J Crit IIIn Inj Sci 2018;8:36-40.

2. Levine MN, Raskob G, Landefeld S, Kearon C. Hemorrhagic complications of anticoagulant treatment. Chest 2001;119(1 Suppl):108S-121S.

3. Wysowski DK, Nourjah P, Swartz L. Bleeding complications with warfarin use: a prevalent adverse effect resulting in regulatory action. Arch Intern Med 2007;167:1414-9.

4. Klein L, Peters J, Miner J, Gorlin J. Evaluation of fixed dose 4-factor prothrombin complex concentrate for emergent warfarin reversal. Am J Emerg Med 2015;33:1213-8.

5. Frumkin K. Rapid reversal of warfarin-associated hemorrhage in the emergency department by prothrombin complex concentrates. Ann Emerg Med 2013;62:616-26.

6. Leissinger CA, Blatt PM, Hoots WK, Ewenstein B. Role of prothrombin complex concentrates in reversing warfarin anticoagulation: a review of the literature. Am J Hematol 2008;83:13743.
7. CSL Behring. Kcentra: prothrombin complex concentrate (human) [Internet]. King of Prussia, PA: CSL Behring [cited 2019 Dec 14]. Available from: http://cslbehring.vo.Ilnwd.net/o33/u/ central/PI/US/Kcentra/EN/Kcentra-Prescribing-Information. pdf.

8. Sarode R, Milling TJ Jr, Refaai MA, et al. Efficacy and safety of a 4-factor prothrombin complex concentrate in patients on vitamin $\mathrm{K}$ antagonists presenting with major bleeding: a randomized, plasma-controlled, phase Illb study. Circulation 2013;128:123443.

9. Astrup G, Sarangarm P, Burnett A. Fixed dose 4-factor prothrombin complex concentrate for the emergent reversal of warfarin: a retrospective analysis. J Thromb Thrombolysis 2018;45:300-5.

10. Scott R, Kersten B, Basior J, Nadler M. Evaluation of fixeddose four-factor prothrombin complex concentrate for emergent warfarin reversal in patients with intracranial hemorrhage. J Emerg Med 2018;54:861-6.

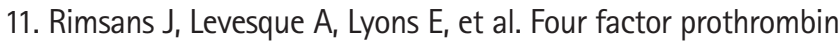
complex concentrate for warfarin reversal in patients with left ventricular assist devices. J Thromb Thrombolysis 2018;46: 180-5.

12. Gilbert EH, Lowenstein SR, Koziol-McLain J, Barta DC, Steiner J. Chart reviews in emergency medicine research: where are the methods? Ann Emerg Med 1996;27:305-8.

13. Kaji $A H$, Schriger $D$, Green $S$. Looking through the retrospectoscope: reducing bias in emergency medicine chart review studies. Ann Emerg Med 2014;64:292-8.

14. Huang WT, Cang WC, Derry KL, Lane JR, von Drygalski A. Fourfactor prothrombin complex concentrate for coagulopathy reversal in patients with liver disease. Clin Appl Thromb Hemost 2017;23:1028-35.

15. Fischer D, Sorensen J, Fontaine GV. Three-factor versus fourfactor prothrombin complex concentrate for the emergent management of warfarin-associated intracranial hemorrhage. Neurocrit Care 2018;28:43-50.

16. Berndtson AE, Huang WT, Box K, et al. A new kid on the block: outcomes with Kcentra 1 year after approval. J Trauma Acute Care Surg 2015;79:1004-8.

17. CSL Behring UK Limited. Beriplex P/N 500 IU [Internet]. Surrey: Datapharm; 2017 [cited 2019 Dec 22]. Available from: https://www.medicines.org.uk/emc/product/6236/smpc.

18. Sin JH, Berger K, Lesch CA. Four-factor prothrombin complex concentrate for life-threatening bleeds or emergent surgery: a retrospective evaluation. J Crit Care 2016;36:166-72.

19. Joseph R, Burner J, Yates S, Strickland A, Tharpe W, Sarode R. Thromboembolic outcomes after use of a four-factor pro- 
thrombin complex concentrate for vitamin $\mathrm{K}$ antagonist reversal in a real-world setting. Transfusion 2016;56:799-807.

20. Jablow LM, Jones CW, Carroll GG, Wilsey SV, Haroz R. Limbthreatening deep venous thrombosis complicating warfarin reversal with three-factor prothrombin complex concentrate: a case report. J Emerg Med 2016;50:28-31.

21. Willis $C M$, Hall $A B$. Use of four-factor prothrombin complex concentrate in the emergency department: a review. J Emerg Nurs 2015;41:9-12. 